\title{
Balancing formal and informal support for
}

\section{psychological health in emergency services:}

\section{creating multiple pathways for ambulance staff}

\author{
Ashlea Kellner, Keith Townsend, Rebecca Loudoun, Tiet-Hanh Dao-Tran and
}

Adrian Wilkinson

It is well accepted that ambulance work is characterised by employee burnout, high stress, work intensification, and exhaustion - physical, mental and emotional (Granter, Wankhade, McCann, Hassard, \& Hyde, 2018). A key factor that contributes to these problems is the unavoidable feature of emergency services work that employees will be regularly exposed to extreme and traumatic events (Bigham et al., 2014; McFarlane \& Bryant, 2007; McFarlane, Williamson, \& Barton, 2009). Exposure of this kind increases the risk of a number of mental health conditions such as depression and anxiety (Izutsu, Tsutsumi, Asukai, Kurita, \& Kawamura, 2004), and post-traumatic stress disorder (Bennett et al., 2005; Grant, Dutton, \& Rosso, 2008; Huizink et al., 2006). There is a significant cost - financial, social and emotional - of mental health conditions to emergency services, to individuals, their friends, families, peers, patients, the community, and the organisation. For emergency services organisations, mental health problems are associated with increased incidence of employee burnout and long term sick leave (Brattberg, 2006), deteriorated health and well-being (Berger et al., 2007) and increased employee turnover (Patterson et al., 2010). It is of increasing importance to emergency services organisations to invest resources in preventing and managing mental health problems. There is also a particular historical culture which can both create stress for individuals while working in contradiction to support mechanisms. For example, many professions that are male-dominated and uniformed tend to have a strict hierarchy, a masculinist 'man up' approach with a culture of blame, fear, bullying and poor industrial 
relations. While these factors appear to be changing with time they are still a major part of the stressful working lives of paramedics.

Research suggests a range of factors can influence the prevalence and severity of consequences associated with trauma exposure and promote positive outcomes. Formal systems of employee support such as access to professional counselling services and resilience training, can positively influence employees' health and wellbeing (Rajaratnam, Sears, Shi, Coberley, \& Pope, 2014; Richmond, Pampel, Wood, \& Nunes, 2017). Informal support via relationships with co-workers and family can be instrumental in reducing the severity of symptoms and encouraging positive post-traumatic growth (Oginska-Bulik, 2015; Prati \& Pietrantoni, 2010; Somville, De Gucht, \& Maes, 2016). Additionally, these formal and informal elements feed into the 'psychosocial safety climate' that can potentially influence employees' physical and psychological state of health (Zadow, Dollard, McLinton, Lawrence, \& Tuckey, 2017).

In this chapter, we examine the formal and informal support systems of two Australian state emergency services cases, with a specific focus on the formal employee assistance and peer support officer programs, and the informal colleague, family, and frontline manager support. Using interview data we explore the components of a balanced support model, and identify strengths and weaknesses of support within the cases. We find these components work together as a network of complementary support functions that can reduce the severity and assist in the management of mental health problems in the ambulance service. A balance of complementary formal and informal support mechanisms can temper the climate and provide a holistic approach to supporting employees that responds to individual preferences. In short we argue that it is not enough to provide formal support systems which function well in their own right. The different needs of staff and the tendency to want the capacity for informal peer support means we need to see the system in terms of how the formal and informal fit together. We need to better undertand how things operate in practice (the interactions of the system in use) rather than the system which is codified on organisational policy documents. Understanding the needs and the actual practice of ambulance service employees when in times of need is critical in improving their work health outcomes. 


\section{Risk factors for mental health problems among emergency responders}

Frontline emergency services workers frequently perform their work in uncontrolled environments that includes exposure to potentially traumatic events (Beaton, Murphy, Johnson, Pike, \& Corneil, 2005; Reichard, Marsh, \& Moore, 2011). Apart from vicarious trauma from exposure to, or involvement in an extreme event, emergency responders are also at high risk of a range of physical incidents that can trigger or lead to mental health problems.

For example, the risk of serious injury for emergency responders is seven times higher than the Australian national average, and the fatality rate is six times higher (Maguire, O'Meara, Brightwell, O'Neill, \& Fitzgerald, 2014). Up to 90 percent of emergency responders are exposed to workplace violence in some form (Pourshaikhian, Abolghasem Gorji, Aryankhesal, Khorasani-Zavareh, \& Barati, 2016); at least half of emergency responders have experienced physical assault (Gabrovec, 2015; Pourshaikhian et al., 2016), and over two thirds have been subject to verbal abuse (Bigham et al., 2014). Additionally, around one in four emergency responders experience sexual harassment or sexual assault on the job (Bigham et al., 2014; Gabrovec, 2015; Pourshaikhian et al., 2016). On the whole, there are a combination of jobrelated risk factors associated with extreme events that present a challenging and at times dangerous work situation for emergency responders. While there is an attempt to influence these factors by public awareness campaigns, the impact of these has been limited.

There are, however, some individual factors and workplace characteristics associated with increased prevalence of PTSD and other psychological conditions that the organisation can exert some degree of control and influence. For instance, pre-trauma risk factors for PTSD include low levels of education (Rybojad, Aftyka, Baran, \& Rzońca, 2016), psychiatric history (Neria, Nandi, \& Galea, 2008), and sleep disturbance (Wright, Britt, Bliese, \& Adler, 2011). Research also suggests that individuals who are younger (Brewin, Andrews, \& Valentine, 2000), female (Skogstad, Heir, Hauff, \& Ekeberg, 2016), and non-married (Berger et al., 2007), are at higher risk of developing PTSD. While some characteristics such as psychiatric history are typically already considered in pre-screening checks, other factors noted here may need further consideration in developing employee support programs that specifically target at risk groups. 
Evidence indicates individual responses to exposure to trauma are difficult to predict and can vary widely. The way individuals cope with trauma may also affect their risk of developing PTSD (Armstrong, Shakespeare-Finch, \& Shochet, 2014; Kirby, Shakespeare-Finch, \& Palk, 2011). For example using maladaptive coping strategies such as avoidant emotional coping (Smith, Drevo, \& Newman, 2017), distraction (Clohessy \& Ehlers, 1999; Sattler, Boyd, \& Kirsch, 2014), rumination, suppression and dissociation (Armstrong et al., 2014; S. Clohessy \& A. Ehlers, 1999) increase the risk of PTSD, hence, the argument that educating employees to understand the difference between adaptive and non-adaptive coping mechanisms is important in helping reduce the risk of mental health disorders.

In the workplace, there are many factors that can contribute to increased rates of PTSD, such as routine work stress (Maguen et al., 2009), organisational stressors (Smith et al., 2017), and employment status (Rybojad et al., 2016). The nature of emergency services work - the types and timing of exposure to traumatic incidents - are also influential. The prevalence of PTSD is higher among those exposed to more types of traumatic events (Skeffington, Rees, \& Mazzucchelli, 2017; Smith et al., 2017), and among those with earlier and more prolonged exposure (Perrin et al., 2007; Sattler et al., 2014).

There are opportunities for employers to address many of these workplace centric factors, for example, by addressing administrative or procedural factors that increase job-related stress, and by improving job security. It is generally unfeasible however, to limit or change factors such as types of trauma individuals are exposed to or the duration of exposure. Rather, emergency services organisations are better served by addressing workplace factors found to reduce the risk of developing psychological conditions or the duration of those conditions, and improve coping skills and post traumatic growth. For example, a strong psychosocial safety climate can buffer the impact of job demand on mental health problems (Hall, Dollard, Winefield, Dormann, \& Bakker, 2013), and the support of colleagues can reduce the incidence of PTSD (Prati \& Pietrantoni, 2010). The focus of this chapter is the provision of support to emergency responders as a means to reduce incidence and better manage psychological conditions associated with trauma exposure. Support, however, is a multifaceted concept; we address it by distinguishing between formal and informal mechanisms. We explore employee 4 | P a g e 
perceptions of their formal support system in two Australian case studies of emergency service organisations and how these operate in balance with the informal support system, underpinned by the psychosocial safety climate.

\section{Formal employee support}

Formal support for employees is often provided under the banner of an Employee Assistance Program (EAP), which is the umbrella term used to capture a range of complementary support services for employees, and sometimes their immediate family members (Scully, 2011). EAPs can be effective through providing preventative health and wellbeing measures, by reducing the incidence of hospitalisation and long-term absences from the workplace, and through improving morale and performance (Richmond et al., 2017). They have been found to have a positive influence on the health and well-being of employees (Rajaratnam et al., 2014; Richmond et al., 2017). Core services in established and well-resourced emergency services organisations include a peer support officer program, 24-hour phone helpline, access to professional counselors and psychologists, debriefing or defusing sessions post-trauma, and education and training (Scully, 2011; Shakespeare-Finch \& Scully, 2008). Access to formal systems of support is critical for emergency response workers to assist them in coping with the trauma that they face.

\section{Informal social support}

In contrast to formal support practices facilitated by an organisation, informal support refers to ad-hoc interactions that can act to 'fill the gaps' of a formal system (Townsend, Wilkinson, \& Burgess, 2013). Social support can be defined as 'the perceived support that helps to meet social needs due to the presence and accessibility of people [that an individual] can trust' (Semerci, 2016: 43). Support can be provided by a number of individuals in the employee's network, including friends, partners, family, colleagues, and supervisors. There is substantial theoretical and empirical evidence that points to social support as a significant contributor to individual well-being (see for instance, Cohen \& Wills, 1985; Halbesleben, 2006; Prati \& Pietrantoni, 2010). 
Social support can improve wellbeing through two key mechanisms (Cohen \& Wills, 1985): it has an immediate buffering effect of lessening the impact when a person is subject to a stressful situation; and accumulation of social resources and integration in a social network acts as a protective mechanism that leads to improved wellbeing over time. These two processes of social support have significant implications for paramedics, reducing immediate and continuing implications of trauma exposure and improving wellbeing and personal growth.

\section{The psychosocial-safety climate}

While the regulatory climate and guidance directed a physical health and safety may be well developed, legislation - and as a result organisations - are less well advanced when it comes to job-related psychological or psychosocial hazards (Zadow et al., 2017). The psychosocial safety climate is characterised by a number of elements that are both tangible and intangible, formal and informal, and is seen as both a precursor to and outcome of employee support systems. Psychosocial safety climate is measured by employees' perceptions about the degree of management commitment and priority given to matters relating to psychological health, and the way the organisation communicates about and encourages participation in these matters (Dollard \& Bakker, 2010; G. B. Hall, Dollard, \& Coward, 2010). Largely, it is a measure of how a combination of factors combine and create an underlying understanding of how psychological health, safety and wellbeing is prioritised and promoted in the organisation. These factors could include formal policies and procedures, managerial behaviour and communication, formal organisational communication, and interactions with other peers and within teams.

There is a growing body of evidence indicating a strong psychosocial safety climate can have significant impact on a range of factors in the workplace, by: reducing the adverse impact of job demands (Bailey, Dollard, McLinton, \& Richards, 2015) and depression (Hall et al., 2013); altering the impact of workplace bullying on PTSD (Bond, Tuckey, \& Dollard, 2010); and, limiting physical and psychological work injury (Zadow et al., 2017). Given the broad potential effect of the psychosocial safety climate, we consider this in our conceptualisation of employee support as a characteristic that underpins the formal and informal elements of 
support. In the remainder of this chapter, we explore the notion of a holistic employee support system in emergency services, examining strengths and weaknesses through the lens of two case studies.

A holistic perspective of employee support

Academic research and findings from our own study suggest both formal and informal support mechanisms are recommended for employees exposed to trauma and stress in the workplace (Beaton, Murphy, Pike, \& Corneil, 1997; Prati \& Pietrantoni, 2010; Scully, 2011). A balance must exist between robust formal mechanisms and strong effective informal mechanisms to ensure individual preferences and situations can be addressed. This concept has been illustrated in Figure 1.

Figure 1. Employee Support System in Emergency Services

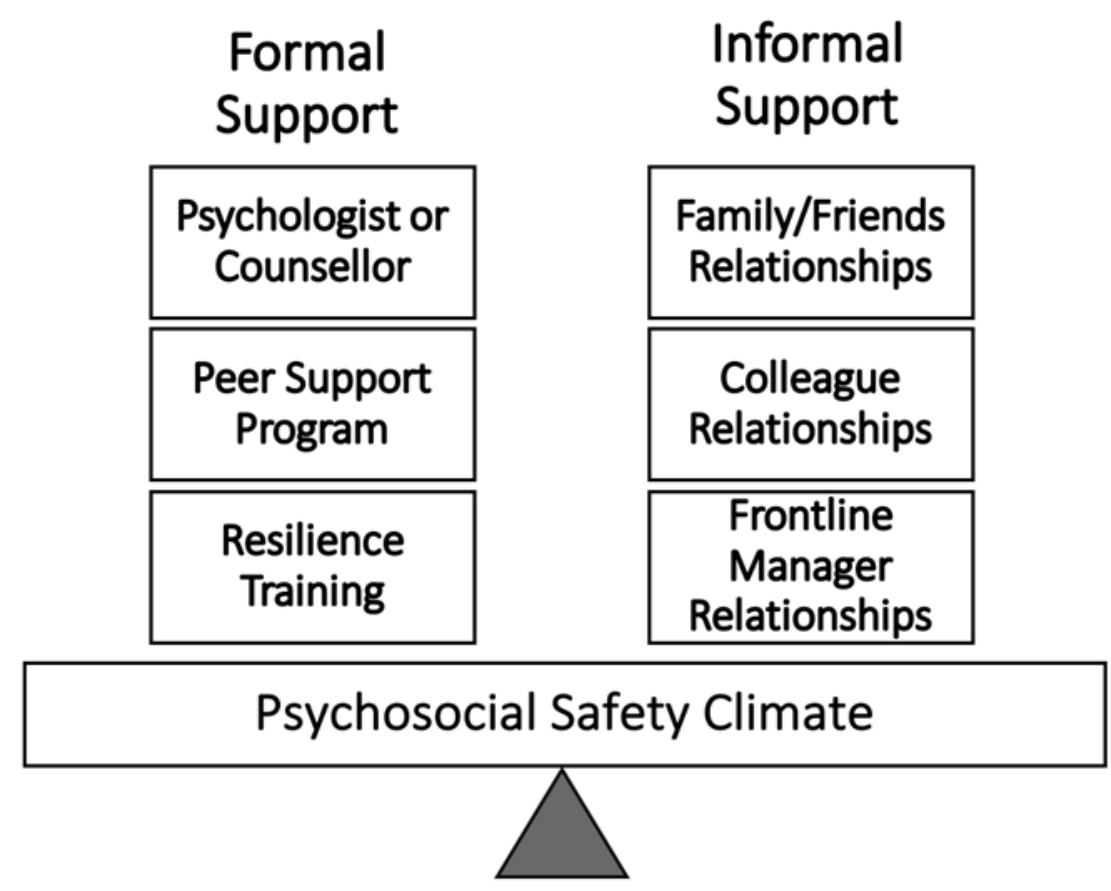

Two Australian cases

Throughout 2015 to 2018 emergency services organisations in three Australian states and territories participated in a research project on the role of, and interactions between people 
management sub-systems and health and well-being outcomes for ambulance employees and ambulance service operations more broadly. The aim of the project was to better understand how these interactions affect outcomes. Specifically, the project explored how the design and management of organisational systems influence employees' individual outcomes such as job satisfaction, intention to quit, and psychological wellbeing including PTSD. The research design combined data collection via phone surveys, interviews, and a range of secondary documents provided by the organisations and unions. While we completed 72 interviews across the three jurisdictions, this chapter reports on findings from the two larger cases only, as data from the third case was toosmall to sensibly generalise and compare (owing to a small population and only ten interviews).

The services both fall under the control of the relevant state or territory government authority. Combined, the services provide assistance to a significant cohort of the Australian population, across metropolitan, rural and extremely remote and indigenous areas. In total they attend around one and a half million cases each year, including emergency and crisis planning and response, pre-hospital patient care, and hospital and inter-facility transport. We provide the cases with the pseudonyms East Service and South Service. The East Service is the largest by area, and employs around 3000 staff, while the South Service has a workforce of around 1000 .

Semi-structured interviews, primarily in person and a small number over the phone, were conducted for the purpose of understanding and explaining key issues and experiences of participants. Seventy-two interviews were conducted with participants ranging from emergency dispatch officers, patient transport officers, paramedics, frontline managers, middle management, upper management, leadership, and union representatives. Interviewees have been numbered following quotes within this chapter, and these numbers correspond with the interviewee details provided in the Appendix. For this chapter, we draw on 62 interviews to illustrate experiences and perceptions of trauma management and mental health support. This number of interviews is at the high end of the range noted by Saunders and Townsend (2016) for interview-based, qualitative research. We make brief mention of the survey results, but do not use that data in detail. The Interviews were audio recorded and professionally transcribed before the content was analysed using a software program, NVivo. 
This data was examined in conjunction with secondary data to identify key themes in the data relating to barriers to provision of employee support. While each interview was completed by a single researcher, the research team met during the data collection process to discuss findings and refine the interview schedule. Analysis began at the start of the interviewing process and concluded after all data were collected. The researchers engaged in an analytical conversation after the interviews to identify preliminary themes that were emerging, to assess the relevance of existing codes to new data, and to examine the relationships between codes (Goetz and LeCompte, 1981). This process fits with the notion of convergent interviewing (Jepson and Rodwell, 2008) which helps improve the internal validity, external and construct validity of qualitative data collection techniques. Interviews were then imported in to the data program NVivo (Version 11) for analysis. Thematic content analysis of interview data resulted in the emergence of themes relevant to the effectiveness of formal and informal employee support.

In both our cases, there was a well-resourced and established formal system of support incorporating all of the elements illustrated in Figure 1. Our findings, however, indicate that informal support from colleagues, family and supervisors still played a central and critical role for employees that couldn't be filled by the formal system. The quality of informal support and the nature of interactions are broadly influenced by the psychosocial safety climate that exists in the organisation. This climate facilitates and influences the strength of the frontline manager and relationships between colleagues that support the employee. A strong climate can thereby alleviate pressure on the support relationships with family and friends, as the employee will have much of their support needs addressed within the workplace environment. Where one or more elements is not present, the balance will begin to tip, and the employees support needs may not be adequately addressed. We explore these aspects of the system through our cases in the following section.

The formal support system

In both cases, significant investment is made to developing robust formalised organisational support systems to assist employee resilience and coping. These services include telephone and face-to-face counselling services, education and training, and a peer support program 
(which is addressed separately and in more detail in the next section). In general, our response from interviewees indicated a positive overall perception of the formal support programs in East Service and South Service. There was a sense that the components of the program were effective individually and fitted together well as a cohesive system. The following interviewee captures the process that followed a critical incident in South Service:

Once an incident has reached a certain point, there's an automatic [process], you have - managers are coming down to do a debrief, the crew's stood down, they all go back to station... I didn't feel completely ready to download to this manager that I'm going to have to sit in front of in future interviews... So the next thing that happened for us was we got a phone call from one of - we have three psychologists in the city that we can access four times a year. So one of those gave us a call that night and that was quite helpful... But then we got follow up from our team leader. 'How's it going? Everything all right?' Just softly, softly. Interview 35 South Service, Paramedic

This quote mentions an initial debrief procedure (formal debriefs are very rarely reported in either case), follow-up phone call from the external counselling service, and informal conversations with the frontline manager. Noted here and in other instances, however, were concerns about future ramifications of having genuine conversations within this support system. This interviewee perceives that having an honest discussion with the manager could compromise their chances of future interviews for promotion, and this was a view echoed in other interviews. For instance:

I feel like we spend a lot of money on our staff welfare through the peer support program and our EAP, the psychologist who we have available to us. But it seems very closed... there's two psychologists that we're allowed to go and see. Now, if you're someone who is thinking I also am applying for this position at the moment and I think this person might be on this panel, I think I just won't go. We're not really given other options. Interview 53 South Service, Frontline Manager 
Many paramedics expressed the view that an authentic conversation about an individual's psychological health within the organisations could later result in a bias that affects career progression. These concerns prohibit some individuals from seeking support within the formal system. Some employees indicated they sought psychological support at their own expense, outside of the organisation, to reduce this risk.

Clearly, the debriefing issue is problematic for not only the organization, but also the staff, and ultimately, the patients. While we can interpret this as the illustration of a lack of care/support for staff, we must also recognise that a lack of openness and a fear by paramedics of seeking out a debrief stems from a long-held legacy of the blame culture. Furthermore, in such a culture there is a lack of opportunity for clinical learning as debriefs are not just about helping the employees exposed to trauma or a stressful event, but they are also clinically important as a learning and reflection exercise.

Another core component of the formal support system is education and training to improve resilience and managerial ability to improve coping. We expected to find a fundamental introduction to resilience and coping skills addressed prior to beginning work, during tertiary education. We conducted an examination of the course topics for the ten universities across East and South Service state areas offering the Bachelor of Paramedic Science degree. Our study found only one university that explicitly advertised an offering of a unit with some content relating to understanding the paramedics own mental health in the context of the work environment. Within this course, only one lecture was dedicated to exploring 'our own mental health and care of the self, mental health continuum'. As the Director of Employee Support in East Service stated, '... what universities offer in that space... is concerning given that the majority of new paramedics do not have the level of life experience once common among ambulance officers in decades past.'

This deficiency in tertiary preparation places pressure on the ambulance service to build resilience and coping skills into their programs. Some resilience training is incorporated into the induction programs in both cases. In South Service and East Service a half day program is provided but the structure of these is not identical. In South Service, the program is a half day 
where managing personal stresses in the workplace, shift work, cumulative stress, and acute stress are discussed. Interviewees in South Service desire a more comprehensive approach to resilience training in the induction and over the course of their career. Paramedics suggest ' $a$ better system is a bit more than a day's training on psychological stressors' [Interview 29, South Service Paramedic]. While clinical training in South Service is generally well resourced, the provision of resilience training is something desired by many employees.

In East Service, a resilience training program has been introduced in recent years as part of the induction process, with positive feedback from employees. This is a four-and-a-half-hour program where participants are provided with resources and activities to complete over the first 12-24 weeks on the road, along with a reflective journal, covering topics such as work life balance, sleep patterns, coping mechanisms, and recognising and managing stress. The program culminates with a visit with an organisational psychologist to 'close the loop'.

East Service also provide a one-day mandatory training program for managers, supervisors and acting supervisors, which focuses on trauma and resilience in the workplace. The program covers legal responsibility for psychological wellbeing, and other topics such as critical incidents, PTSD, anxiety, depression and suicide. Over a period of almost ten years the program appears to impact of the way managers and supervisors interact with employees around psychological wellness, as demonstrated by the following example of an exercise that has been conducted in the program since its inception.

We say to them, what's all the worst things you could say when [paramedics] get back or when they come back to the station? It used to be that there was lots of energy in the room. People would be recounting what had been said to them in the past. There would be a whole heap of things that people would come up with. Interestingly, I really saw a contrast last year. The same scenarios. When you ask them what's all the things that would be helpful, the room would go quiet and you wouldn't hear anything. But last year it was the opposite way around. It struck me because it was the biggest contrast I've seen around a group of managers. All the 'worst things'... They were having trouble trying to think of things! And 'what are 
all the helpful things?' The room got active and they were - they came up with loads of things! Interview 65 East Service, Director Employee Support

As the previous quote illustrates, there has been a sustained effort to deliver practical skills to help managers conduct positive interactions about mental health and wellness with their employees. Over time, the introduction of this program has reshaped managerial interactions with employees about mental health and support. It is possible that development of similar skills among employees would enable them to provide enhanced support to colleagues - who our research suggests are the preferred source of informal support for many participants.

\section{The peer support program}

The peer support program aims to provide employees with early intervention and support following exposure to an incident. Through a number of pathways, employees exposed to critical and traumatic incidents can contact, or are contacted by, a peer support officer (PSO) soon after exposure to the incident. PSOs are carefully selected and trained in areas such as counselling skills, components of acute stress, psychological trauma, bereavement, suicide and effective communication skills. Typically, the PSO will contact an employee by phone after receiving notification from a centralised service that exposure to particularly traumatic incident such as suicide or paediatric death has occurred.

The PSO program is well established across East and South Service. Our data indicates the program is generally viewed positively by employees although some elements of the system are seen as frustrating. For example, the timeframe in which a PSO contacts the paramedics, the means of communication, and the integrity of the conversations were identified as potential areas of concern.

Firstly, the period of time between an incident occurring and contact from a PSO was a topic of mixed feelings. While some interviewees prefer time to process the event before speaking about it with a PSO, others believe contact should be made sooner. For instance, a South Service paramedic recounted a call over a day after an incident was like 'a slap in the face because it was a significant job the day before in the morning, but over 24 hours later you get a phone call... I couldn't hang up quick enough' [Interview 43]. The timing of PSO contact is quite nuanced and differs based on the individual preferences. The following interviewee 
provides a detailed suggestion for an alternative model of contact that allowed the individual to exert more control to shape the contact to their needs:

I think we need to empower people to use the program and not necessarily enforce the program on people. 'Hi, this is Doug doing a peer support call.' 'I'm fine, thanks. Thanks for the phone call. Appreciate it. See you later.' Is that the right way? I think we need to actually now start talking to our staff and saying, 'what type of peer support do you want?' I think that's where we could use technology to our advantage. So you could generate an automatic message going out. So as soon as peer support is activated, bang, 'hey, Doug, peer support's been activated. Do you need to speak to somebody now? (a) No? (b) Yes.' Bang, it fires back to the person who's doing the peer support activation. Peer support required now. Interview 32 South Service, Paramedic

While the program is seen as generally effective, more flexibility in how it engages with employees and caters to their support preferences for contact type and timing would be beneficial. This may be through an auto-generated or personalised text message very soon after a critical incident occurs. Such an approach is likely to cater to a subset of employees who indicated that they do not engage with PSOs, but the contact is still important. This is because it is not the two-way communication that is important, but the acknowledgement of the difficult job they have attended. 'I feel better that somebody cares, that Comms [The Communications Department] recognised that I had to do something difficult' [Interivew 7, East Service Paramedic]. Hence, in some cases, it is not the timing that is particularly important but the contact itself, which may have the same effectiveness in a different format such as an initial text message which gives the employee control of whether and how they choose to respond to support.

Paramedics suggest that the use of a text message - although it can be viewed as impersonal -gives the employee more discretion to make contact at a time that they can ensure they are able to talk privately and when they are ready. The following paramedic discusses this technique as applied by a PSO: 
She'll send you a very gentle text and she'll follow it up in a week's time... So what I like about the texts is ... if you need me, if you want to - and sometimes - that's the only time I've actually reacted to a PSO and texted back, you know, actually it was an awful job. Interview 45 South Service, Paramedic

Overall, there was a small but notable degree of concern that employees exposed to major trauma could occasionally 'fall through the cracks' when they are not contacted in a reasonable time period (or at all). This is where the interaction and balance between the formal systems of support and the informal means of support become critical.

Informal support from colleagues and family

Informal support is complementary to the formal system in place in these organisations. In our survey, we found that most employees drew support from colleagues and family, and the least from frontline managers. Social support from colleagues and family can be effective at addressing the emotional and informational components of support. Support from colleagues, we found, is valuable for revisiting cases and exploring what happened and the clinical actions taken by the employee. Among each other, colleagues can discuss the technical aspects of often quite traumatic situations to better understand and process the event. This type of support usually cannot be provided by friends and family, who, according to our respondents, are better suited to discussing issues relating to the working conditions or other related stressors.

Our interviews indicated there was high importance placed by staff on the informal interactions by paramedics in the ambulance vehicle or at the hospital following a traumatic event. This casual discussion, often involving 'black humour', replaces the need for formal debrief and allows employees to process information and relieve the state of hyperarousal.

Colleague support is fundamental for assisting coping in employees exposed to critical incident stress. The scope of training seems to centre upon self-care, rather than caring for work colleagues and identifying signs and symptoms of stress-related mental illness, and how to respond and provide support to those suffering. 
'You might do a case that's very challenging... All you really want to do is speak to your peers... to go over how you went professionally' [Interview 34, South Service Paramedic].

The role of peers is vital as it fills a space that generally cannot be filled by close friends and family who are less familiar with the trauma associated with emergency service work. As another paramedic summarized, 'You can't go home and tell people, I had a baby die today... then they become needy' [Interview 34, East Service Paramedic].

As these excepts demonstrate, family are not always a suitable sounding board for discussing difficult cases - peers are critical for this aspect of support. The difficulty associated with peer support however is the high workload experienced by many employees, particularly in the metropolitan areas. There is very limited down-time between cases, so 'there's no chance to defuse after a job because they're bouncing you from job to job, you never get the chance to process what just happened because you're now onto the next event' [Interview 11, East Service Clinical Educator]. There can also be a great deal of inconsistency between the peers rostered on in the same vehicle, which - particularly in larger stations - can lead to challenges forming lasting relationships.

Informal support from frontline managers

In our survey, over half of respondents indicated they receive no or little support from their frontline manager. Low frontline manager support was also noted to be related to higher incidence of PTSD and intention to quit, and lower job satisfaction in our study. As has been shown in a vast array of previous research, frontline managers play a key role in the experiences of employees (Hutchinson and Purcell 2010, Townsend et al 2011), hence understanding why frontline managers are not viewed as a primary source of support is important in further research. Certainly FLMs can and do play a formal role, however we focus here on the informal role that they can play supporting their employees.

Interviewees suggested some key barriers to the provision of informal social support by frontline managers. These generally related to the frontline manager's attitude (particularly 
their expressed empathy), and their workload as it impacts availability for support. First, issues relating to mindset and attitude are best described by an employee:

Oh, at [a city station] I had two team leaders, because I split between the two [stations], and they were polar opposites. One was very helpful and supportive. The other one was an elitist and didn't have any personal skills. Didn't know how to communicate with people. Apparently - well, not apparently - gave off the feeling he just didn't care. Yeah, it was like polar opposites. Interview 41 South Service, Paramedic

While this response was not unanimous, there was a cohort of employees that expressed perceived deficiencies in frontline managers' core support skills such as empathy, concern, and genuine compassion. This may be a result of a promotional process that does not adequately consider the emotional intelligence and skillset of frontline managers. Alternately, it may be that frontline managers are not all receiving the degree of development needed to provide the type of support that each individual requires.

Another key theme in our data was that the workload or location of the frontline manager was a major hurdle in the provision of adequate support. There is frustration because FLMs 'might see [their team] at the start of a shift but they don't get access to the staff because of workload' [Interview 2, East Service Paramedic]. These issues of workload and availability seem to be compounded in some stations where FLMs are also required to spend a period of their day on the road responding to calls. As one regional manager explains:

Their job was meant to be a team leader, to look after the staff. It wasn't to be a clinician. That was the sort of thing they were going to use if last resort, call these guys for backup if there's no one else. But they have used them and abused them and used them as a resource to treat patients. But in doing that, they now don't do their job properly as a team leader. Interview 71 East Service, Regional Manager

While at times the lack of FLM presence at stations means support cannot be accessed, clearly the seriousness of this issue varies by geographical location. In small regional and rural stations, so FLMs staff interaction can be limited because of time spent driving long distances 
between remote towns. Other FLMs in regional locations are allocated to work on the road and are rarely in the station themselves. In busy metropolitan stations, the FLM can be subject to such a heavy workload that they are unable to meet with staff particularly where teams are very large. Some FLMs in metropolitan stations in the East service reported that - as staff here do not return to the station between jobs - it can be impossible to have face to face contact unless FLMs visit the nearby hospital and catch employees on the ramp. There is however, substantial variation and no clear answers that apply consistently across the organisation.

\section{Toward a balanced support system}

First responders are subject not only to regular trauma exposure but a range of work conditions that can create a high level of stress in their everyday lives (Granter et al., 2018; Sterud, Hem, Ekeberg, \& Lau, 2008). To achieve the highest level of reliability and safety in the services provided to the community, first responders must operate in an environment where they are adequately supported. For one employee, suitable support may be a chat with a peer support officer following a traumatic incident. For another, it may be a discreet visit to the psychologist, or to defuse with friends after work at the pub. The preferred support pathway will change, with time and personal preference, according to the type of trauma exposure, or due to other personal events occurring concurrently. Support preferences are changeable, and they are personal. As noted there is a particular historical culture in the service which can both create stress and mitigate against support. Consequently, changing the system is required. The system (formal and informal interacting) must evolve to be more open and supportive while recognising that systems of support need to align to the workplace as it currently stands and not as it ought to be.

Our research offers messages that will be of value to other emergency service organisations. The formal support systems need to maintain the utmost degree of integrity and separation from management and other processes. If employees are to utilise the system they must feel confident it is a confidential process and that interaction with it will not be detrimental to their career. Where an aversion to using the formal mechanisms develop among staff, this 
will place undue pressure on the informal mechanisms, and the support system will become unbalanced.

There are some potential improvements to processes and programs of the formal system that are evident from our case studies. Currently, in Australia, resilience training in tertiary studies for emergency responders is limited or non-existent. The responsibility for building employee resilience rests with the organisation. We cannot overstate the significance and value of high quality initial (during the induction process) and ongoing resilience training for employees, to better enable identification of mental health conditions in themselves and others, and provide tools to assist coping. Additionally, there is added value in developing these programs to extend, where possible, to the greater network of employees' family and friends, given the importance identified of this support in our research and that of others (Evans, Pistrang, \& Billings, 2013).

The support system exists in the context of the psychosocial safety climate of the organisation. To strengthen this component of support, leaders must act to destigmatise conversations around psychological health, and to model positive behaviours of self-care, peer support, and facilitating post-traumatic growth. Team leaders and frontline managers are critical here, and support can be improved by developing managerial attitude and mindset, and addressing physical availability to employees. While emotional support needs appear to be addressed by family, friends, and - to a degree - colleagues, the frontline manager is key in providing the advice, direction and resources that aid recovery and coping. The learning here relates to balance; to minimise employee suffering and promote positive growth there must be equilibrium between formal and informal support mechanisms available to staff, resting on the psychosocial safety climate. Individual support needs are not identical, and they change over time, hence a robust system with multiple support pathways is the most desirable process.

\section{References}

Armstrong, D, Shakespeare-Finch, J, \& Shochet, I. (2014). 'Predicting post-traumatic growth and post-traumatic stress in firefighters', Australian Journal of Psychology, 66: 1, 3846. 
Bailey, TS, Dollard, MF, McLinton, SS, \& Richards, PAM. (2015). 'Psychosocial safety climate, psychosocial and physical factors in the aetiology of musculoskeletal disorder symptoms and workplace injury compensation claims', Work \& Stress, 29: 2, 190-211.

Beaton, R, Murphy, S, Johnson, C, Pike, K, \& Corneil, W. (2005). 'Exposure to duty-related incident stressors in urban firefighters and paramedics', J Trauma Stress, 11: 4, 821828.

Beaton, R, Murphy, SA, Pike, KC, \& Corneil, W. (1997). 'Social support and network conflict in firefighters and paramedics', West J Nurs Res, 19: 3, 297-313.

Bennett, P, Williams, Y, Page, N, Hood, K, Woollard, M, \& Vetter, N. (2005). 'Associations between organizational and incident factors and emotional distress in emergency ambulance personnel', British Journal of Clinical Psychology, 44: 2, 215-226.

Berger, Figueira, I, Maurat, AM, Bucassio, EP, Vieira, I, Jardim, SR, et al. (2007). 'Partial and full PTSD in Brazilian ambulance workers: prevalence and impact on health and on quality of life', J Trauma Stress, 20: 4, 637-642.

Bigham, BL, Jensen, JL, Tavares, W, Drennan, IR, Saleem, H, Dainty, KN, et al. (2014). 'Paramedic Self-reported Exposure to Violence in the Emergency Medical Services (EMS) Workplace: A Mixed-methods Cross-sectional Survey', Prehospital Emergency Care, 18: 4, 489-494.

Bond, SA, Tuckey, MR, \& Dollard, MF. (2010). 'Psychosocial Safety Climate, Workplace Bullying, and Symptoms of Posttraumatic Stress', Organization Development Journal, 28: 1, 37-56.

Brattberg, G. (2006). 'PTSD and ADHD: underlying factors in many cases of burnout', Stress and Health, 22: 5, 305-313.

Brewin, CR, Andrews, B, \& Valentine, JD. (2000). 'Meta-analysis of risk factors for posttraumatic stress disorder in trauma-exposed adults', J Consult Clin Psychol, 68: 5, 748-766.

Clohessy, \& Ehlers. (1999). 'PTSD symptoms, response to intrusive memories and coping in ambulance service workers', Br J Clin Psychol, 38, 251-265.

Clohessy, S, \& Ehlers, A. (1999). 'PTSD symptoms, response to intrusive memories and coping in ambulance service workers', British Journal of Clinical Psychology, 38: 3, 251-265.

Cohen, S, \& Wills, TA. (1985). 'Stress, social support, and the buffering hypothesis', Psychological bulletin, 98: 2, 310.

Dollard, MF, \& Bakker, AB. (2010). 'Psychosocial safety climate as a precursor to conducive work environments, psychological health problems, and employee engagement', Journal of Occupational and Organizational Psychology, 83: 3, 579-599.

Evans, R, Pistrang, N, \& Billings, J. (2013). 'Police officers' experiences of supportive and unsupportive social interactions following traumatic incidents', European Journal of Psychotraumatology, 4: 1, 19696.

Ferry, FR, Brady, SE, Bunting, BP, Murphy, SD, Bolton, D, \& O'Neill, SM. (2015). 'The Economic Burden of PTSD in Northern Ireland', J Trauma Stress, 28: 3, 191-197.

Gabrovec, B. (2015). 'The prevalence of violence directed at paramedic services personnel', Obzornik Zdravstvene Nege, 49: 4, 284-294.

Grant, AM, Dutton, JE, \& Rosso, BD. (2008). 'Giving Commitment: Employee Support Programs and the Prosocial Sensemaking Process', Academy of Management Journal, 51: 5, 898-918. 
Granter, E, Wankhade, P, McCann, L, Hassard, J, \& Hyde, P. (2018). 'Multiple Dimensions of Work Intensity: Ambulance Work as Edgework', Work, Employment and Society, 0950017018759207.

Halbesleben, JR. (2006). 'Sources of social support and burnout: a meta-analytic test of the conservation of resources model', Journal of applied Psychology, 91: 5, 1134.

Hall, Dollard, M, Winefield, A, Dormann, C, \& Bakker, A. (2013). 'Psychosocial safety climate buffers effects of job demands on depression and positive organizational behaviors', Anxiety, Stress, \& Coping, 26: 4, 355-377.

Hall, GB, Dollard, MF, \& Coward, J. (2010). 'Psychosocial Safety Climate: Development of the PSC-12', International Journal of Stress Management, 17: 4, 353-383.

Huizink, AC, Slottje, P, Witteveen, AB, Bijlsma, JA, Twisk, JW, Smidt, N, et al. (2006). 'Long term health complaints following the Amsterdam Air Disaster in police officers and firefighters', Occup Environ Med, 63: 10, 657-662.

Hutchinson, S. and Pucell, J. (2010) 'Managing ward managers for roles in HR in the NHS: overworked and under-resourced', Human Resource Management Journal, Vol.20, No.4, 357-74.

Ivanova, JI, Birnbaum, HG, Chen, L, Duhig, AM, Dayoub, EJ, Kantor, ES, et al. (2011). 'Cost of post-traumatic stress disorder vs major depressive disorder among patients covered by medicaid or private insurance', Am J Manag Care, 17: 8, e314-323.

Izutsu, T, Tsutsumi, A, Asukai, N, Kurita, H, \& Kawamura, N. (2004). 'Relationship between a traumatic life event and an alteration in stress response', Stress and Health, 20: 2, 6573.

Kirby, R, Shakespeare-Finch, J, \& Palk, G. (2011). 'Adaptive and Maladaptive Coping Strategies Predict Posttrauma Outcomes in Ambulance Personnel', Traumatology, 17: 4, 25-34.

Maguen, S, Metzler, T, McCaslin, S, Inslicht, S, Henn-Haase, C, Neylan, T, et al. (2009). 'Routine work environment stress and PTSD symptoms in police officers', The Journal of Nervous and Mental Disease, 197: 10, 754-760.

Maguire, BJ, O'Meara, PF, Brightwell, RF, O'Neill, BJ, \& Fitzgerald, GJ. (2014). 'Occupational injury risk among Australian paramedics: an analysis of national data', Med J Aust, 200 : $8,477-480$.

McFarlane, \& Bryant. (2007). 'Post-traumatic stress disorder in occupational settings: anticipating and managing the risk', Occup Med, 57: 6, 404-410.

McFarlane, Williamson, \& Barton. (2009). 'The impact of traumatic stressors in civilian occupational settings', J Public Health Policy, 30: 3, 311-327.

Neria, Y, Nandi, A, \& Galea, S. (2008). 'Post-traumatic stress disorder following disasters: a systematic review', Psychological Medicine, 38: 04, 467-480.

Oginska-Bulik, N. (2015). 'Social support and negative and positive outcomes of experienced traumatic events in a group of male emergency service workers', Int J Occup Saf Ergon, 21: 2, 119-127.

Patterson, PD, Jones, CB, Hubble, MW, Carr, M, Weaver, MD, Engberg, J, et al. (2010). 'The longitudinal study of turnover and the cost of turnover in EMS', Prehospital emergency care : official journal of the National Association of EMS Physicians and the National Association of State EMS Directors, 14: 2, 209-221.

Perrin, MA, DiGrande, L, Wheeler, K, Thorpe, L, Farfel, M, \& Brackbill, R. (2007). 'Differences in PTSD prevalence and associated risk factors among World Trade Center disaster rescue and recovery workers', Am J Psychiatry, 164: 9, 1385-1394. 
Pourshaikhian, M, Abolghasem Gorji, H, Aryankhesal, A, Khorasani-Zavareh, D, \& Barati, A. (2016). 'A Systematic Literature Review: Workplace Violence Against Emergency Medical Services Personnel', Archives of Trauma Research, 5: 1, e28734.

Prati, G, \& Pietrantoni, L. (2010). 'The relation of perceived and received social support to mental health among first responders: a meta-analytic review', Journal of Community Psychology, 38: 3, 403-417.

Rajaratnam, AS, Sears, LE, Shi, Y, Coberley, CR, \& Pope, JE. (2014). 'Well-being, health, and productivity improvement after an employee well-being intervention in large retail distribution centers', Journal of occupational and environmental medicine, 56: 12, 1291-1296.

Reichard, AA, Marsh, SM, \& Moore, PH. (2011). 'Fatal and nonfatal injuries among emergency medical technicians and paramedics', Prehospital Emergency Care, 15: 4, 511-517.

Richmond, MK, Pampel, FC, Wood, RC, \& Nunes, AP. (2017). 'The impact of employee assistance services on workplace outcomes: Results of a prospective, quasiexperimental study', Journal of occupational health psychology, 22: 2, 170.

Rybojad, B, Aftyka, A, Baran, M, \& Rzońca, P. (2016). 'Risk Factors for Posttraumatic Stress Disorder in Polish Paramedics: A Pilot Study', The Journal of emergency medicine, 50 : 2, 270-276.

Sattler, DN, Boyd, B, \& Kirsch, J. (2014). 'Trauma-exposed Firefighters: Relationships among Posttraumatic Growth, Posttraumatic Stress, Resource Availability, Coping and Critical Incident Stress Debriefing Experience', Stress and Health, 30: 5, 356-365.

Saunders, M., and Townsend, K., (2016) 'Reporting and Justifying the Number of Interview Participants in Organisation and Workplace Research' British Journal of Management, 27 (4): 836-852.

Scully, PJ. (2011). 'Taking care of staff: A comprehensive model of support for paramedics and emergency medical dispatchers', Traumatology, 17: 4, 35-42.

Semerci, A. (2016). 'The effect of social support on job stress of entrepreneurs', Academy Of Entrepreneurship Jounal, 22: 1, 41.

Shakespeare-Finch, JE, \& Scully, PJ. (2008). 'Ways in which paramedics cope with, and respond to, natural large-scale disasters', in, The Phoenix of Natural Disasters: Community Resilience: Nova Science.

Skeffington, PM, Rees, CS, \& Mazzucchelli, T. (2017). 'Trauma exposure and post-traumatic stress disorder within fire and emergency services in Western Australia', Australian Journal of Psychology, 69: 1, 20-28.

Skogstad, L, Heir, T, Hauff, E, \& Ekeberg, $\varnothing$. (2016). 'Post-traumatic stress among rescue workers after terror attacks in Norway', Occupational Medicine, 66: 7, 528-535.

Smith, RJ, Drevo, S, \& Newman, E. (2017). 'Covering traumatic news stories: Factors associated with post-traumatic stress disorder among journalists', Stress and Health, n/a-n/a.

Somville, FJ, De Gucht, V, \& Maes, S. (2016). 'The impact of occupational hazards and traumatic events among Belgian emergency physicians', Scandinavian journal of trauma, resuscitation and emergency medicine, 24: 1, 59.

Sterud, T, Hem, E, Ekeberg, $\varnothing, \&$ Lau, B. (2008). 'Occupational stressors and its organizational and individual correlates: a nationwide study of Norwegian ambulance personnel', $B M C$ emergency medicine, 8: 1, 16.

Townsend, K., Wilkinson, A. and Allan, C. (2012) 'Mixed signals in HRM: the HRM role of hospital line managers', Human Resource Management Journal, Vol. 22, No.3, 267-82. 
Townsend, K, Wilkinson, A, \& Burgess, J. (2013). 'Filling the gaps: Patterns of formal and informal participation', Economic and Industrial Democracy, 34: 2, 337-354.

Wright, KM, Britt, TW, Bliese, PD, \& Adler, AB. (2011). 'Insomnia severity, combat exposure and mental health outcomes', Stress and Health, 27: 4, 325-333.

Zadow, AJ, Dollard, MF, McLinton, SS, Lawrence, P, \& Tuckey, MR. (2017). 'Psychosocial safety climate, emotional exhaustion, and work injuries in healthcare workplaces', Stress and Health, 558-569. 